\title{
Ciência das políticas públicas: reflexões sobre sua diversidade analítica*
}

\author{
Luis F. Mota (https://orcid.org/ https://orcid.org/0000-0003-0618-8553)' \\ Instituto Politécnico de Leiria, Leiria, Portugal.
}

Resumo: O objetivo deste ensaio é apresentar uma revisão sobre as principais abordagens analíticas que têm caracterizado a ciência das políticas públicas, bem como apresentar uma reflexão crítica sobre a diversidade analítica da disciplina em termos teóricos, epistemológicos, metodológicos e até de influências disciplinares. De igual modo, procede-se a uma reflexão sobre os principais debates presentes e futuros da disciplina.

Desta análise ressalta a enorme diversidade que caracteriza esta disciplina, que tem, inclusivamente, dado origem a diferentes comunidades de analistas, com radicais disciplinares e objetivos de análise bem diversos. De igual modo, torna-se evidente que as diferentes abordagens seguem as tendências gerais das ciências sociais com debates entre positivistas e pós-positivistas, bem como entre teóricos defensores da primazia da agência ou da estrutura na explicação da mudança e continuidade das políticas públicas.

Palavras-chave: Ciência das políticas públicas. Processo de políticas públicas. Teorias. frameworks de análise. Metodologias.

\section{Policy sciences: reflections on its analytical diversity}

Abstract: The purpose of this essay is to present a review of the main analytical approaches that have characterized Policy Sciences, as well as to present a critical reflection on the analytical diversity of this discipline regarding theoretical, epistemological, methodological, and even disciplinary influences. Likewise, one aims to present a reflection on the main present and future debates of the discipline.

This analysis highlights the enormous diversity that characterizes this discipline, which has even given rise to different communities of analysts, with very different disciplinary backgrounds and goals of analysis. Likewise, it becomes evident that the different approaches of this discipline follow the general tendencies of Social Sciences with debates between positivists and post-positivists, as well as among theoreticians who advocate the primacy of agency or structure in explaining the change and continuity of public policies.

Keywords: Policy sciences. Policy-making process. Theories. frameworks of analysis. Methodologies.

\author{
* Este artigo foi \\ desenvolvido com \\ o apoio de bolsa de \\ pós-doutoramento \\ da Fundação \\ para a Ciência e \\ Tecnologia (SFRH/ \\ BPD/115117/2016) \\ de que $o$ autor \\ usufruía aquando da \\ redação da primeira \\ versão deste artigo. \\ $\mathrm{O}$ autor gostaria \\ de agradecer à \\ doutora Maria \\ Engrácia Cardim \\ e aos revisores e \\ editores desta revista \\ pelos comentários a \\ versões preliminares \\ deste artigo.
}

I. Luis F. Mota é professor adjunto de administração pública no Instituto Politécnico de Leiria, Leiria, Portugal, e pesquisador na unidade de pesquisa em governança, competitividade e políticas públicas (Govcopp) da Universidade de Aveiro. Tem pósdoutorado em políticas públicas pela Universidade de Aveiro e é doutor em administração pública pela Universidade de Lisboa. <luismota.work@ gmail.com>. 


\section{Introdução}

ciência das políticas públicas é um ramo das ciências sociais que, apesar
de ser bastante recente, se caracteriza por uma significativa diversidade de
abordagens analíticas. Esta diversidade deve-se, de acordo com Peters e Pierre (2006), à complexidade e multidimensionalidade do seu objeto de estudo. No âmbito de tal complexidade, um dos aspetos que mais importa reconhecer é o facto de o processo de elaboração de políticas públicas em geral ser considerado não apenas como exercício técnico e racional de definição de objetivos e alocação de meios inerentes à intervenção sobre um problema, mas também como resultado de exercícios de interação entre diferentes atores (Peters \& Pierre, 2006; Howlett, Ramesh \& Perl, 2020).

A perspetiva dinâmica e interativa inerente a esta concepção mais alargada do estudo de políticas públicas, apesar de ser mais abrangente, comporta, contudo, maior dificuldade de análise (Sabatier, 2007). Neste sentido, Sabatier (2007) e Peters e Pierre (2006) consideram que será compreensível (ou até inevitável) que os analistas de políticas públicas adotem estratégias de simplificação analítica, nomeadamente através do desenvolvimento de uma multiplicidade de abordagens de análise, suportadas em diferentes teorias, estratégias metodológicas e influências disciplinares.

Tendo por base este cenário de complexidade, o objetivo deste artigo é apresentar uma revisão e reflexão crítica sobre os principais debates e abordagens que têm caracterizado a investigação em políticas públicas nas últimas décadas. Considera-se

1. Recorde-se, a este propósito, que as frameworks de análise têm como principal objetivo ajudar a identificar as variáveis mais importantes no estudo de um fenómeno, não especificando, habitualmente, as relações expectáveis entre variáveis tal como feito pelas teorias, sendo, portanto, suficientemente abrangentes para abarcar diferentes teorias sobre um mesmo fenómeno (Ostrom, 2007). este exercício necessário na medida em que os analistas de políticas públicas têm abordagens bastante diversas, o que faz com que nem todos conheçam - e reconheçam como válidas - as bases teóricas e as ferramentas metodológicas uns dos outros. Ademais, considera-se essencial a produção de mais textos em língua portuguesa sobre a temática do processo de políticas públicas, visto que o seu estudo ainda se encontra pouco desenvolvido ao nível teórico em países como o Brasil ou Portugal. Tal situação deve-se, em larga medida, ao facto de a disciplina da ciência das políticas públicas só ter conhecido alguma institucionalização no Brasil já no século XXI (Farah, 2016), o mesmo se passando em Portugal (Tavares, 2006), ao contrário do que se passou nos Estados Unidos e na Europa Central, que desenvolveram a disciplina no último quartel do século XX.

No sentido de cumprir o objetivo referido, este artigo começa por apresentar uma breve resenha do percurso de emergência e evolução da ciência das políticas públicas. Em segundo lugar, serão apresentadas as principais teorias e frameworks ${ }^{1}$ de 
análise utilizadas no estudo do processo de políticas públicas. Seguidamente, serão examinadas criticamente as principais abordagens analíticas da ciência das políticas públicas, com uma reflexão sobre as principais diferenças epistemológicas, teóricas e metodológicas. O artigo será concluído com algumas notas sobre quais os principais debates presentes e futuros da disciplina.

\section{Emergência e afirmação da ciência das políticas públicas ao nível internacional: uma breve resenha histórica}

Como DeLeon e Vogenbeck (2007) referem num ensaio sobre a história da ciência das políticas públicas, esta disciplina é caracterizada por uma curta história, mas por um longínquo passado, uma vez que existem políticas públicas e aconselhamento político desde os primórdios da história, em especial desde o período renascentista. Apesar de muito importantes para o nascimento da disciplina da filosofia política, estas práticas de aconselhamento político não deverão ser confundidas com a disciplina da ciência das políticas públicas, na medida em que não eram habitualmente baseadas em investigação científica e eram frequentemente pouco imparciais ou objetivas (DeLeon \& Martell, 2006).

Os verdadeiros antecedentes da ciência das políticas públicas remontam, assim, apenas ao período do final do século XIX e início do século XX, durante o qual se assistiu ao desenvolvimento de diversas ciências sociais e da investigação empírica aplicada, a uma maior consciência em relação aos problemas sociais, bem como ao desenvolvimento de uma linha de pensamento filosófico de orientação pragmática e instrumental nos Estados Unidos (DeLeon \& Vogenbeck, 2007).

É no contexto histórico acima descrito que surgem os primeiros apelos ao desenvolvimento de uma "policy orientation" das ciências sociais, inicialmente pela mão de autores como Charles Merriam, Robert Lynd ou Robert Merton, tal como reconhecido por Harold Lasswell (1951), por muitos considerado o pai da ciência das políticas públicas. Estes foram, assim, alguns dos autores que influenciaram Lasswell (1951) em sua proposta de desenvolvimento de uma nova disciplina das ciências sociais, que deveria estar estruturada em torno de três características:

i. um carácter multidisciplinar, que "rompesse" com as divisões disciplinares existentes;

ii. uma orientação para os problemas, criando uma ciência social aplicada e com pendor mais pragmático; 
iii. e explicitamente normativa, advogando que nenhum problema social estaria incólume de elementos valorativos, uma vez que lida com relações sociais e interpessoais.

Muito embora a proposta de Lasswell seja hoje reconhecida como o início da ciência das políticas públicas, importa referir que os apelos do autor e de seus antecessores terão encontrado algumas resistências iniciais, sobretudo relacionadas com a proposta de orientação para a ciência aplicada (John, 2013; Cairney \& Weible, 2017). Com efeito, a efetiva afirmação da ciência das políticas públicas nos Estados Unidos só viria a dar-se a partir de meados da década de 1960, na sequência do crescimento do designado Welfare State nos Estados Unidos e uma crescente aposta governamental no planeamento e avaliação de grandes programas públicos, bem como pela decorrente criação de programas de ensino e investigação e de associações e jornais científicos dedicados a esta disciplina (Sabatier, 1991; Hill \& Hupe, 2014).

Os primeiros analistas a abraçar a "policy orientation" terão sido, de acordo com DeLeon e Vogenbeck (2007), académicos das áreas da investigação operacional e da economia, os quais se dedicavam, sobretudo, à avaliação de políticas e que defendiam que os problemas e soluções inerentes aos processos de elaboração de políticas poderiam ser definidos de forma compreensiva e racional e submetidos a análise quantitativa. Muito embora esta "vaga" de investigação em avaliação de políticas tenha contribuído para o desenvolvimento da disciplina, dada a sua preocupação com o rigor metodológico, importa registar que também terá conduzido a algum ceticismo quanto à sua aplicabilidade, por se ter revelado pouco sensível a variáveis políticas e necessidades dos decisores políticos (DeLeon \& Vogenbeck, 2007).

Foi com base nas críticas a esta abordagem - que alguns designam por "policy analysis" ("análise de políticas públicas"), como será explicado adiante - que surgiu outra corrente de investigação apelidada de "policy studies" ("estudos de políticas públicas"), que atribui maior importância aos processos, contextos e a outras variáveis políticas. Foi precisamente neste âmbito que se assistiu a um reforço da utilização da "framework do ciclo de políticas públicas", que divide o processo de políticas públicas em diferentes fases, nomeadamente identificação dos problemas e agendamento, formulação, tomada de decisão, implementação e avaliação (Jann \& Wegrich, 2007; Parsons, 1995). De igual modo, destacaram-se como um claro exemplo desta abordagem os designados "estudos de implementação", que surgiram na década de 1970 e tiveram o seu auge no início da década seguinte e que deram um importante contributo complementar para a disciplina ao se debruçarem mais sobre a análise dos processos, embora gerando também bastante confusão analítica (DeLeon \& Vogenbeck, 2007; Hill \& Hupe, 2014; Mota, 2020). 
Em face do ceticismo e da confusão analítica descrita, prevalecia nos Estados Unidos, na década de 1980, um sentimento de ceticismo quanto à utilidade prática da ciência das políticas públicas. Esta situação devia-se às dúvidas quanto à credibilidade da disciplina, mas também ao aumento dos movimentos de defesa de "redução do Estado" e da abordagem da gestão pública, a qual tem uma perspetiva mais organizacional, em detrimento da perspetiva sistémica da ciência das políticas públicas (DeLeon \& Vogenbeck, 2007).

As décadas de 1970 e 1980 foram igualmente marcadas, de acordo com Parsons (1995), pela afirmação da ciência das políticas públicas para além das fronteiras dos Estados Unidos, sobretudo em países como o Reino Unido, a Alemanha ou os Países Baixos $^{2}$, muito embora tenha encontrado algumas resistências iniciais e tenha gerado alguma desilusão pouco tempo depois, devido ao excessivo foco economicista. Os principais desenvolvimentos da ciência das políticas públicas na Europa terão sido, de facto, no âmbito da abordagem dos estudos de políticas públicas, nomeadamente no estudo da implementação, sobretudo numa perspetiva multiator, mas também nos métodos participativos de análise de políticas públicas (Parsons, 1995).

No início da década de 1990, Paul Sabatier (1991), um dos mais notáveis autores da ciência das políticas públicas, reconhecia que a disciplina havia percorrido um assinalável percurso de evolução nas décadas anteriores. No entanto, apresentava algumas críticas, a principal das quais prendendo-se à falta de produção teórica, sobretudo no âmbito da linha de estudos focados no conteúdo de políticas setoriais, havendo, portanto, a necessidade de se desenvolverem melhores teorias (Sabatier, 1991).

Na quarta secção deste ensaio proceder-se-á à análise dos principais contributos teóricos e analíticos das últimas décadas, sendo, assim, possível aferir se o apelo de Sabatier se concretizou. Antes disso, será necessário perceber quais os principais elementos de análise das políticas públicas, bem como as principais teorias clássicas que estiveram na base das frameworks e teorias específicas sobre o processo de políticas públicas mais recentes.

\section{Elementos de análise e teorias de mudança do processo de políticas públicas}

A ciência das políticas públicas tem sido particularmente profícua em diferentes abordagens analíticas nas últimas décadas, as quais estão focadas em diferentes elementos do processo de políticas públicas. A este propósito, importa destacar, desde logo, que o estudo e análise das políticas não se centra apenas nos instrumentos de políticas públicas (policy content) e nos seus produtos, resultados e impactos (policy

\footnotetext{
2. Os exemplos mais claros dessa tendência serão as obras pioneiras de Michael Hill, no Reino Unido, de Fritz Scharpf ou de Benny Hjern e seus associados, na Alemanha, e de um vasto conjunto de escolas nos Países Baixos.
} 
outputs, outcomes and impacts), mas também na análise das estruturas responsáveis pelo desenho e implementação da política (polity) e no processo de interação entre diversos tipos de atores que tentam influenciar a tomada de decisões aos mais diversos níveis (politics)ou ainda nos diferentes determinantes que influenciam o processo (policy determinants) (Howlett, Ramesh, \& Perl, 2020; Knill \& Tosun, 2020).

Ao nível do estudo das estruturas de políticas públicas (polity), será importante destacar, desde logo, os estudos relacionados com os papéis de cada ator de políticas públicas - para uma visão global, ver Howlett, Ramesh e Perl (2020), Knill e Tosun (2020) ou Kingdon (2010). Para além desses estudos, é ainda importante diferenciar entre os conceitos de "universo de políticas públicas" ("policy universe"), que compreende todos os atores que estão, de alguma forma, relacionados com um domínio de políticas e o conceito de "subsistema de políticas" ("policy subsystem"), que contempla apenas os atores que têm capacidade efetiva de influenciar a formulação de políticas nesse domínio (Howlett, Ramesh, \& Perl, 2020).

Na esfera do estudo do conteúdo das políticas (policy content), poder-se-á realçar, por exemplo, as diferentes tipologias de instrumentos de políticas, das quais se logrará destacar a apresentada por Christopher Hood (1986), que procedeu à distinção de quatro tipos de instrumentos, aos quais estão associados diferentes recursos: informação, autoridade, financiamento e organização.

Apesar da mais-valia dos estudos sobre os atores e o conteúdo das políticas públicas, o domínio em que a produção científica de teorias e ferramentas de análise tem sido mais profícua é a referente ao estudo do processo político, notadamente aos determinantes que conduzem a dinâmicas de mudança e estabilidade em políticas públicas.

A propósito de mudanças em políticas públicas, importa, desde logo, destacar o trabalho de Hall (1993), que distinguiu três grandes tipos de mudanças: as de calibração dos instrumentos (como a mudança na fórmula de cálculo de um benefício); as de alteração nos instrumentos (como a contratualização externa de funções anteriormente disponibilizadas por serviços públicos); e as de paradigmas (como a despenalização do aborto).

Na análise das teorias relacionadas à mudança em políticas públicas, importa referir, primeiramente, que grande parte das teorias atuais têm subjacentes outras teorias organizacionais mais antigas, das quais Knill e Tosun (2020) destacam as seguintes:

i. abordagem racional, enunciada por autores como Lasswell, entre outros, que assume que o processo de políticas públicas segue uma lógica racional de resolução de problemas; 
ii. abordagem da racionalidade limitada, enunciada por autores como Herbert Simon ou James March, que manifesta que o processo de tomada de decisão decorre num contexto de racionalidade, apesar da necessária simplificação das escolhas disponíveis, resultante de condicionalismos de acesso e processamento de informação; e,

iii. incrementalismo, enunciado por autores como Charles Lindblom, que rejeita a ideia de que o processo de políticas públicas segue uma lógica puramente racional e que tem em conta variáveis políticas, nomeadamente o nível de contestação e de tecnicidade dos problemas, o que conduz à tendência para decisões pouco disruptivas.

Com base nas abordagens teóricas mais antigas referidas tem surgido, nas últimas décadas, um amplo conjunto de teorias que se foca em diferentes variáveis-chave, tais como as instituições, os grupos de interesse e as redes, os fatores socioeconómicos de nível macro, o comportamento (racional) de atores individuais ou ainda as ideias (John, 2013; Heikkila \& Cairney, 2018). A este propósito, Knill e Tosun (2020) destacam a existência de três grandes grupos de abordagens teóricas:

- teorias baseadas nas estruturas, das quais destacam, primeiramente, a Escola Socioeconómica, que explica a mudança nas políticas públicas pela evolução dos desenvolvimentos sociais e económicos. Adicionalmente, destacam também a abordagem das clivagens, que refere que as políticas públicas dependem de escoIhas societais, as quais estão fundadas num conjunto de clivagens ideológicas (centro $\times$ periferia; Estado $\times$ Igreja; rural $\times$ urbano; trabalhadores $\times$ empresários; materialismo $\times$ pós-materialismo; e, sociedades abertas $\times$ fechadas);

- teorias baseadas nas instituições, das quais destacam, por um lado, o institucionalismo clássico, com a sua abordagem formal-legal, que se debruça sobre o papel desempenhado pelos diferentes organismos públicos e as diferentes formas de funcionamento do sistema político (democracias maioritárias $\times$ consensuais; estilos de políticas de acordo com famílias de nações etc.). Por outro lado, destacam ainda o neoinstitucionalismo sociológico, que refere que as escolhas individuais em políticas públicas estão fortemente relacionadas com as culturas institucionais e as interpretações existentes dos problemas e das soluções que são percepcionadas como apropriadas ou ainda o neoinstitucionalismo histórico, que refere que as escolhas de políticas públicas da atualidade são condicionadas por escolhas e padrões de interação passados;

- teorias baseadas nos interesses individuais, das quais se destacam as teorias da racionalidade e da racionalidade limitada já referidas, bem como a teoria da escolha pública, a teoria dos jogos 
e o institucionalismo de escolha racional. Por um lado, a teoria da escolha pública assume que o comportamento individual dos atores (políticos, burocratas e eleitores) é guiado pelo autointeresse e pela maximização da sua satisfação. Por sua vez, a teoria dos jogos refere que a atuação de um ator depende sempre da atuação dos restantes. Por último, o institucionalismo de escolha racional refere que as escolhas dos indivíduos seguem uma lógica de "consequencialismo", na medida em que decorrem da ponderação dos incentivos externos negativos e positivos e das oportunidades disponibilizadas no contexto das instituições.

Para além do enfoque num conjunto limitado de variáveis e unidades de análise, outra estratégia de simplificação bastante comum tem sido o recurso à utilização de frameworks de análise e de teorias específicas sobre o processo de políticas públicas, tal como será abordado no próximo ponto deste ensaio.

\section{Principais frameworks e teorias sobre o processo de políticas públicas}

Uma das primeiras frameworks do estudo do processo de políticas públicas foi a designada "framework do ciclo de políticas públicas" (ou "framework estagista"), que analisa a elaboração de políticas públicas como um processo que se divide num conjunto de fases inter-relacionadas, cuja sequência segue uma lógica de resolução de problemas e nas quais participam um diferenciado número de atores (Jann \& Wegrich, 2007; Howlett, Ramesh, \& Perl, 2020):

i. identificação de problemas e seu agendamento ao nível público e político, no âmbito da qual podem participar um vastíssimo conjunto de atores interessados num determinado domínio de políticas (universo de políticas públicas), tais como partidos, meios de comunicação, think-tanks, cidadãos, atores políticos, grupos de interesse etc.;

ii. formulação de propostas de intervenção sobre os problemas e seu debate e análise, na qual habitualmente só participa um conjunto mais restrito de atores (subsistema de políticas) com poderes oficiais e de grupos de interesse organizados;

iii. tomada de decisão sobre a(s) medida(s) a adotar, que está reservada aos atores políticos que têm poder oficial para tomar decisões;

iv. implementação da(s) medida(s), no âmbito da qual participam o aparelho administrativo do Estado ou organismos dos setores privado e não lucrativo que estabeleceram contratos ou parcerias com o Estado; e 
v. avaliação da execução das intervenções, aferindo a qualidade de processos, resultados e impactos da execução das intervenções, que pode ser feita por um vasto conjunto de atores, com maiores ou menores graus de cientificidade ou senso comum.

Apresentada pela primeira vez, em 1956, por Lasswell, a "framework do ciclo de políticas públicas" conheceu desenvolvimentos e reformulações ao longo da década de 1970 fortemente influenciadas pela abordagem sistémica de Easton ${ }^{3}$ (Jann \& Wegrich, 2007). Muito embora possa ser considerada uma representação mais realista do processo de políticas públicas e tenha sido praticamente incontestada durante as décadas de 1960 e 1970, esta framework tem sido alvo de críticas, nomeadamente por apresentar uma concepção demasiado simplista e "estilizada" do processo (Jann \& Wegrich, 2007; Sabatier, 2007). Apesar dessas críticas, a "framework do ciclo de políticas públicas" será ainda uma das mais renomadas e utilizadas, por ser uma ferramenta analítica que possibilita a redução da complexidade do processo de políticas públicas num conjunto de fases ou subfases, cada uma das quais podendo ser investigada em separado, com questões analíticas, hipóteses e teorias parciais próprias (Jann \& Wegrich, 2007; Sabatier, 2007).

Um conjunto de frameworks e teorias apresentadas como alternativas à "framework do ciclo de políticas públicas", desde a década de 1980, centram-se, aliás, em apenas algumas fases do ciclo de políticas públicas, nomeadamente no agendamento, formulação e tomada de decisão ${ }^{4}$. Poder-se-ão destacar neste âmbito a "framework dos fluxos múltiplos", a "teoria do equilíbrio pontuado", a "framework das coligações de interesses" (advocacy coalition framework) ou a "framework de análise e desenvolvimento institucional".

A "framework dos fluxos múltiplos", apresentada por John Kingdon em 1984, no seu livro Agendas, alternatives, and public policies, dedica-se ao estudo dos processos de agendamento e formulação de alternativas, sobretudo em domínios de políticas com elevada ambiguidade, através de uma perspetiva sistémica. Entre outras ideias defendidas, Kingdon (2010) advoga que esses processos não ocorrem ordenadamente por fases, mas sim em três fluxos relativamente independentes:

i. fluxo dos problemas, no qual se definem quais os problemas que ganham maior notoriedade pública, influenciados por mecanismos de concentração de atenção, tais como a apresentação de indicadores, a ocorrência de eventos-foco e a disponibilização de feedbacks de políticas existentes;
3. Em sua abordagem, Easton (1965) defendia que o processo político deveria ser entendido como um sistema que interage com o seu contexto/ ambiente, recebendo inputs, notadamente exigências e manifestações de apoio, e produzindo outputs, que incluem as decisões, bem como as ações necessárias à implementação dessas decisões, os quais influenciam novamente os inputs, através de processos de feedback.

4. A explicação para menor interesse por fases como a implementação advém da menor atenção que esta tem merecido desde a década de 1990 devido à derivação para temas mais elementares da gestão pública. Para uma explicação deste fenómeno e para uma revisão das frameworks mais antigas e mais recentes da fase de implementação, leiase Hill \& Hupe (2014) ou, em português, Mota (2020). 
ii. fluxo político, composto por fatores políticos independentes da identificação de problemas e propostas de soluções, tais como resultados eleitorais, mudanças no governo ou distribuição partidária e ideológica do órgão legislativo; e,

iii. fluxo das (propostas de) políticas públicas, no qual comunidades de especialistas (da academia e da administração) criam, debatem e (re) formulam propostas de solucionamento de problemas, cuja aceitação depende de sua exequibilidade técnica e operacionalidade orçamental, de sua aceitação política e pública e de sua congruência com valores dominantes.

Segundo Kingdon (2010), estes fluxos têm uma existência relativamente independente entre si, até que se abram "janelas de oportunidade" - na sequência de um acontecimento político relevante, tal como uma mudança de governo ("political window") ou de uma manifestação evidente de um problema, como uma catástrofe ("problem window") -, no âmbito dos quais defensores de uma determinada proposta de política previamente desenhada (os designados policy entrepreneurs) tentam ativamente que esta seja adotada. Apesar de conceituada, esta framework tem sido alvo de algumas críticas que advogam, sobretudo, que os fluxos não serão assim tão independentes entre si, já que, por exemplo, as comunidades de especialistas não são apolíticas e que a abertura de "janelas de oportunidade" não será assim tão aleatória.

A "teoria do equilíbrio pontuado", apresentada originalmente por Frank Baumgartner e Bryan Jones, no livro Agendas and instability in American politics, de 1993, é, de igual modo, uma heurística dedicada às fases do agendamento e formulação, na qual os autores defendem que estes processos sejam conduzidos habitualmente numa lógica incremental, sendo ocasionalmente abalados por mudanças abruptas.

De acordo com os autores desta teoria, a estabilidade de um determinado domínio de políticas públicas é assegurada quando existe uma certa hegemonia de pensamento em relação ao problema e às possíveis propostas de intervenção sobre o mesmo ("policy image") (Baumgartner, Jones, \& Mortensen, 2018). De igual modo, a estabilidade é mantida pelos designados "subsistemas", compostos por um grupo relativamente estável e próximo de pessoas especialistas no domínio de políticas, os quais também podem ser designados por "policy monopolies", quando prevalece uma "ideia" hegemónica sobre a política pública (Baumgartner, Jones, \& Mortensen, 2018). A este propósito, importa ainda distinguir os subsistemas e os macrossistemas, sendo estes últimos compostos pelos líderes dos governos, os quais têm uma abordagem mais genérica e seletiva aos problemas políticos (Baumgartner, Jones, \& Mortensen, 2018). 
Em face das condições referidas, as mudanças abruptas num determinado domínio de políticas públicas apenas ocorrem quando surgem, no âmbito do subsistema, "imagens" alternativas quanto ao problema e, sobretudo, quanto às propostas exequíveis para lidar com o mesmo, mas também quando esse "assunto" migra dos subsistemas (mais orientados para mudanças incrementais) para o macrossistema (Baumgartner, Jones, \& Mortensen, 2018). As maiores limitações desta heurística são admitidas pelos próprios autores, que reconhecem a incapacidade de prever quando irá ocorrer a mudança, bem como a sua intensidade ou as mudanças que irá provocar (Baumgartner, Jones, \& Mortensen, 2018).

A "framework das coligações de interesses" é, por sua vez, uma proposta de Paul Sabatier, formulada em 1988, no artigo "An advocacy coalition framework of policy change and the role of policy-oriented learning therein" e que mais tarde foi desenvolvida no livro intitulado Policy change and learning: an advocacy coalition approach, em coautoria com Hank Jenkins-Smith. De acordo com esta heurística, os subsistemas de domínios de políticas mais complexos e contestados tendem a dar origem à criação de "coligações de interesses". Cada coligação de interesses é composta por diversos atores, oficiais e não oficiais, que compartilham um conjunto de valores e crenças sobre os problemas e as soluções de intervenção adequadas, os quais são baseados em suas convicções profundas e mobilizam seus recursos para defender seus próprios "pontos de vista", ao mesmo tempo em que tentam descredibilizar as coligações oponentes (Jenkins-Smith et alii, 2018).

Para os autores, a mudança na política pública pode ser determinada por mudanças em "fatores externos" que influenciam o funcionamento das coligações ou por "fatores internos". Na lista de fatores externos encontram-se eventos externos ao subsistema (mudanças em condições socioeconómicas, na opinião pública, na coligação governativa e no funcionamento de outros subsistemas), bem como mudanças na estrutura de oportunidade da coligação (grau de consenso necessário para mudanças, abertura do sistema político e clivagens societais) ou até em parâmetros relativamente estáveis (atributos básicos do problema e distribuição de recursos naturais, valores socioculturais fundamentais e estrutura social e a estrutura constitucional) (Jenkins-Smith et alii, 2018). Em relação aos "fatores internos", destacam-se os choques internos que provoquem mudanças no entendimento dominante do problema, os acordos negociados ou a aprendizagem política resultante de processos de negociação, nos quais a utilização de informação técnica poderá desempenhar um papel fundamental (Jenkins-Smith et alii, 2018).

As principais limitações desta framework são apontadas por seus próprios autores e referem-se à dificuldade de aplicação, dado que exigem um estudo longitudinal, bem 
como à não aplicabilidade a domínios de políticas que não se caracterizem por uma clara demarcação de posições entre diferentes coligações (Jenkins-Smith et alii, 2018).

Para além destas três frameworks que se focam, sobretudo, ao nível dos subsistemas, merece também um particular destaque a "framework de análise e desenvolvimento institucional", desenvolvida por Elinor Ostrom e seus colaboradores durante a década de 1980, num amplo conjunto de publicações, e que tem sido amplamente utilizada para o estudo de domínios de políticas que implicam a gestão comum de bens públicos. Nesta framework, que usa a abordagem do institucionalismo de escolha racional, as políticas públicas são concebidas como "arranjos institucionais" de ação coletiva, ou seja, conjuntos de regras coletivas que permitem ou constrangem ações individuais de nível operacional, as quais podem, no entanto, ser mudadas pelos próprios indivíduos (Ostrom, 2007). As mudanças de políticas resultam, assim, da conjugação de ações estratégicas de diferentes indivíduos, que tentam mudar os "arranjos institucionais" para melhorar as suas circunstâncias (Ostrom, 2007).

Nesta framework, Ostrom e os seus colaboradores consideram que a designada "arena de ação" é influenciada por fatores macro como os atributos da comunidade, condições físicas e materiais da envolvente e, sobretudo, regras institucionais de nível constitucional, no qual são definidos as regras e os procedimentos de distribuição de poder (Ostrom, 2007). Esta "arena de ação" é composta por atores com os seus atributos (nomeadamente os seus valores e recursos), mas também pela "situação da ação" - ou seja, o problema em questão -, cuja análise inclui os seguintes fatores:

(1) um conjunto de participantes, (2) as posições específicas que serão preenchidas por participantes, (3) o conjunto de ações permitidas e a sua ligação com os efeitos, (4) os efeitos potenciais que estão ligados a sequências de ação individuais, (5) o nível de controlo que cada participante tem sobre a escolha, (6) a informação disponível para os participantes sobre a estrutura da situação da ação e (7) os custos e benefícios - que operam como incentivos e dissuasores - associados a ações e efeitos (Ostrom, 2007).

Os efeitos desta "arena de ação" são, por sua vez, também influenciados pelos "padrões de interação", que podem ter uma natureza mais egoísta ou cooperativa. Para Heikkila e Cairney (2018), a principal vantagem desta framework é o facto de não fazer nenhuma assunção específica sobre o comportamento humano ou sobre as instituições, já que é a conjugação de um conjunto de indivíduos e de normas que determina se os indivíduos se comportam de forma egoísta ou cooperativa na produção de bens públicos. 
Para além destas quatro frameworks já amplamente consagradas na investigação em políticas públicas existe ainda um conjunto de outras que têm sido apontadas como promissoras no estudo do processo de políticas públicas nas duas últimas edições do livro Theories of the policy process (Sabatier \& Weible, 2014; Weible \& Sabatier, 2018): a framework da construção social, desenvolvida por Helen Ingram e Anne Schneider, que defende que o desenho das políticas públicas é amplamente influenciado pela construção social em relação aos seus grupos-alvo predominante na opinião pública e entre os decisores políticos, premiando aqueles que são vistos como dignos e penalizando aqueles que são percepcionados como indigentes; a framework das narrativas das políticas, proposta por Elizabeth Shanahan, Mark McBeth e Michael Jones, que analisa a utilização de narrativas sobre as origens, os objetivos e os impactos esperados de determinada política, sobretudo por parte de coligações no âmbito de subsistemas; a teoria do feedback de políticas (policy feedback theory), desenvolvida por Suzanne Mettler e seus colaboradores, que, adotando a assunção que as políticas públicas moldam a política, tenta perceber o que acontece após a adoção das políticas, sobretudo na esfera de sua interpretação na opinião pública; e os modelos de inovação e difusão, amplamente estudados por Frances Berry e William Berry, que identificaram que os principais mecanismos pelos quais se processa a difusão de inovações entre jurisdições são a aprendizagem, a competição e a pressão para a conformidade em face dos padrões definidos por organismos de nível superior.

Como se pode observar, existem diversas teorias e frameworks que se propõem a analisar o processo de políticas, desde as mais antigas, que são mais holísticas e menos densas analiticamente, até às mais recentes, que procedem a análises mais focadas, embora mais circunscritas a fenómenos específicos. Com efeito, a ciência das políticas públicas tem sido particularmente profícua em diferentes abordagens, as quais têm diferentes enfoques, mas também distintos radicais teóricos e metodológicos e até disciplinares ${ }^{5}$. Estas diferenças serão alvo de análise mais aprofundada na próxima secção.

\section{Diversidade epistemológica, metodológica e disciplinar da ciência das políticas públicas}

A ciência das políticas públicas é, como referido, uma disciplina caracterizada por uma significativa diversidade analítica, ainda que o início de sua afirmação científica tenha ocorrido há apenas cerca de cinco décadas. As diferentes estratégias analíticas utilizadas para estudar políticas públicas não diferem, contudo, apenas quanto aos pontos de enfoque, apresentando igualmente diferentes pressupostos
5. Para uma análise comparativa mais aprofundada das frameworks referidas no artigo, leia-se Heikkila \& Cairney (2018). 
epistemológicos, bases disciplinares, estratégias metodológicas e até comunidades analíticas.

A presença de tais diferenças esteve, desde logo, subjacente à emergência das abordagens da "análise de políticas públicas" e dos "estudos de políticas públicas", anteriormente referidas, cujas diferenças foram reconhecidas, desde logo, pelo próprio Lasswell (1951) e por outros autores (vide, inter alia, Howlett, Ramesh \& Perl, 2020; DeLeon \& Vogenbeck, 2007). Ver Quadro 1:

- por um lado, a abordagem da análise de políticas públicas é maioritariamente adotada por analistas que trabalham para organismos estatais ou grupos de interesse e que tendem a desenvolver análises formais, utilizando princípios da economia, sobretudo do ramo da economia do bem-estar, através de estudos com pressupostos positivistas, os quais se baseiam em análises concretas dos objetivos e impactos das políticas; e, por outro lado,

- a abordagem dos estudos de políticas públicas, que é maioritariamente desenvolvida por académicos, os quais desenvolvem frequentemente estudos com base em pressupostos pós-positivistas mais próximos da ciência política, que se debruçam sobre a interpretação dos objetivos, as intenções e as ações dos atores envolvidos nas diferentes etapas do processo de políticas.

QUADRO 1

DISTINÇÃO ENTRE AS ABORDAGENS

"ANÁlise de políticAs" e "estudos de políticas"

\begin{tabular}{|c|c|c|}
\hline & $\begin{array}{l}\text { Análise de políticas públicas } \\
\text { (policy analysis) }\end{array}$ & $\begin{array}{l}\text { Estudos de políticas públicas } \\
\text { (policy studies) }\end{array}$ \\
\hline Principal objetivo & $\begin{array}{l}\text { Melhorar o desenho, implementação } \\
\text { e avaliação das políticas existentes } \\
\text { (conhecimento no processo) }\end{array}$ & $\begin{array}{l}\text { Compreender o processo de elaboração de } \\
\text { políticas públicas e produzir teoria } \\
\text { (conhecimento do processo) }\end{array}$ \\
\hline Principal objeto de estudo & $\begin{array}{l}\text { Instrumentos, resultados e } \\
\text { impactos das políticas }\end{array}$ & Processo de elaboração das políticas \\
\hline Principal assunção & $\begin{array}{l}\text { Problemas e soluções vistos } \\
\text { de forma racional e otimizada }\end{array}$ & $\begin{array}{l}\text { Problemas e soluções como resultado } \\
\text { de acordos e negociações plurais }\end{array}$ \\
\hline $\begin{array}{l}\text { Princípio metodológico } \\
\text { subjacente }\end{array}$ & Procura de respostas mais universais & Atenção ao contexto \\
\hline $\begin{array}{l}\text { Princípio epistemológico } \\
\text { subjacente }\end{array}$ & $\begin{array}{l}\text { Positivismo } \\
\text { (analisa as atividades efetivas } \\
\text { das organizações) }\end{array}$ & $\begin{array}{l}\text { Interpretativismo } \\
\text { (estuda os objetivos e intenções } \\
\text { subjacentes às decisões) }\end{array}$ \\
\hline $\begin{array}{l}\text { Principal disciplina de } \\
\text { inspiração }\end{array}$ & Economia e investigação operacional & Ciência política e ciências do comportamento \\
\hline
\end{tabular}


Como é possível observar pelo descrito, é possível distinguirem-se ainda diferentes inspirações disciplinares na multiplicidade de abordagens no âmbito da ciência das políticas públicas. Poder-se-ão destacar, a este propósito, as diferenças de contributos da ciência política, da economia e da sociologia, com reflexo ao nível dos pressupostos de análise, da principal unidade de análise e das fases do processo de políticas mais analisadas (Pierre, 2006), conforme o Quadro 2. De acordo com Pierre (2006), os contributos destas três disciplinas organizam-se nos seguintes moldes:

- ciência política: concentra-se primordialmente na disputa de poder entre diferentes atores, quer sejam indivíduos, grupos ou organizações, focando-se, sobretudo, nas fases do agendamento e tomada de decisão, sendo geralmente criticada pela sua tendência exacerbada em centrar a sua análise nas elites;

- economia: tem geralmente como assunção a racionalidade de comportamento dos atores individuais e tende a focar-se na análise comparativa das diferentes opções políticas ou na avaliação das políticas, através da análise da eficiência e eficácia das intervenções desenvolvidas, sendo geralmente criticada pela dificuldade em incorporar variáveis políticas, tais como o conflito político ou a inércia institucional;

- sociologia: tende a concentrar-se no estudo dos problemas sociais e na aferição dos impactos das políticas públicas na mitigação desses problemas, bem como na análise dos contextos organizacionais das estruturas implementadoras, focando-se, por isso, nas fases da identificação de problemas, implementação e avaliação de políticas. É geral-

\section{QUADRO 2}

PRINCIPAIS INFLUÊNCIAS DISCIPLINARES DA CIÊNCIA DAS POLÍTICAS PÚBLICAS

\begin{tabular}{|c|c|c|c|}
\hline & Ciência política & Economia & Sociologia \\
\hline Principal enfoque & $\begin{array}{l}\text { a "Luta" de poder entre } \\
\text { atores (indivíduos, grupos e } \\
\text { instituições) }\end{array}$ & a Comportamento individual & $\begin{array}{l}\text { a Problemas sociais que } \\
\text { originaram as políticas } \\
\text { a Efeitos das políticas } \\
\square \text { Contextos organizacionais }\end{array}$ \\
\hline Fase mais analisada & $\begin{array}{l}\text { agendamento } \\
\text { 口 Tomada de Decisão }\end{array}$ & $\begin{array}{l}\text { • Escolha dos instrumentos } \\
\text { • Avaliação (eficiência e } \\
\text { eficácia) }\end{array}$ & $\begin{array}{l}\text { • Identificação do problema } \\
\text { • Implementação } \\
\text { • Avaliação (impacto) }\end{array}$ \\
\hline Principais críticas & $\begin{array}{l}\text { Dificuldade em integrar } \\
\text { indivíduo e estrutura num } \\
\text { mesmo modelo } \\
\text { a Enfoque exacerbado das } \\
\text { elites }\end{array}$ & $\begin{array}{l}\text { Dificuldade em incorporar } \\
\text { conflito político e inércia } \\
\text { institucional }\end{array}$ & $\begin{array}{l}\text { a Limitado interesse pela } \\
\text { tomada de decisão } \\
\text { a Considera que relações } \\
\text { poder são definidas por eco- } \\
\text { nomia e status social }\end{array}$ \\
\hline
\end{tabular}


mente criticada pelo limitado interesse pelos processos de tomada de decisão ou por considerar que as relações de poder são definidas por variáveis relacionadas com a economia ou o status social.

Para além dos aspetos já referidos, importa destacar ainda que a ciência das políticas públicas contemporânea se baseia quer no método dedutivo, no qual os analistas aplicam pressupostos ou princípios gerais a fenómenos específicos (teoria da escolha pública, teoria de classes ou o neoinstitucionalismo de escolha racional), quer no método indutivo, cujas generalizações resultam de análises empíricas de fenómenos e posterior teste com comparação com outros casos (neoinstitucionalismos sociológico e histórico ou teorias pluralistas ou corporativistas) (Howlett, Ramesh \& Perl, 2020).

Ainda no domínio dos métodos utilizados, Heikkila e Cairney (2018) dão conta de uma enorme diversidade de desenhos de pesquisa no estudo do processo de políticas com recurso às frameworks e das teorias acima referidas - por um lado, a "framework dos fluxos múltiplos", a "framework da construção social" e a "teoria do feedback de políticas" baseiam-se, maioritariamente, em estudos de caso; por outro lado, a "teoria do equilíbrio pontuado" baseia-se, sobretudo, em dados quantitativos de séries temporais; e, por outro lado ainda, a "framework de coligações de interesse" e a "framework das narrativas das políticas" utilizam desenhos mistos, com conjugação de questionários, entrevistas e análise documental.

Como é possível observar, a ciência das políticas públicas está envolta numa enorme diversidade de perspetivas analíticas, as quais dão, por vezes, origem a conclusões bastante díspares, o que tem contribuído para aumentar a (percepção de) complexidade desta disciplina. A convivência entre estas diferentes abordagens constitui-se, portanto, em um dos principais desafios para o futuro da ciência das políticas públicas, que são alvo de análise na última secção deste ensaio.

\section{Debates presentes e futuros da ciência das políticas públicas}

Tal como foi possível observar ao longo do artigo, a ciência das políticas públicas caracteriza-se por uma particular complexidade, a qual advém não apenas da multidimensionalidade do seu objeto de estudo, mas ainda da profusão de estratégias analíticas existentes.

No âmbito desta diversidade analítica, destaca-se, desde logo, a existência de diferentes correntes teóricas que focam distintas variáveis enquanto determinantes 
mais importantes na explicação da mudança e estabilidade no processo de políticas públicas, nomeadamente instituições, grupos e redes, fatores exógenos, atores individuais racionais e ideias (John, 2013).

Muito embora essas abordagens teóricas apresentem significativas diferenças entre si, importa referir que as mesmas não são necessariamente incompatíveis, já que traduzem perspetivas referentes a diferentes níveis e enfoques de análise, sendo, por isso, importante que qualquer investigador explicite devidamente a abordagem utilizada, por forma a evitar "falsos debates teóricos" (Knill \& Tosun, 2020). Tal como referem Knill e Tosun (2020), as abordagens com enfoque nas "instituições" e nos "interesses individuais" demonstram, por exemplo, algumas complementaridades entre si, na medida em que a primeira é especialmente adequada para a análise de padrões mais amplos de mudança nas políticas públicas, ao passo que a última permite a compreensão de escolhas concretas. A emergência de frameworks e teorias sintéticas que conjugam diferentes abordagens constitui-se, portanto, como um exercício particularmente importante para o avanço da ciência das políticas públicas - algumas das frameworks mais recentes destacadas na secção anterior são, de acordo com Ayres e Marsh (2013), exemplos dessa mesma possibilidade.

Apesar da desejabilidade do estabelecimento de ligações entre diferentes abordagens, Ayres e Marsh (2013) referem que importa ter em atenção que tal esforço pode ser inviável, na medida em que essas teorias têm princípios ontológicos subjacentes que nem sempre são compatíveis entre si. As abordagens ontológicas das diferentes abordagens teóricas têm, de facto, sido responsáveis por alguns dos principais debates atuais na ciência das políticas públicas, havendo uma clara disputa entre positivistas e pós-positivistas. Sempre de acordo com Ayres e Marsh (2013), no centro do debate entre essas duas correntes ontológicas está o "monismo metodológico", ou seja, a (in)desejabilidade da aproximação das ciências sociais relativamente às ciências naturais em termos metodológicos - por um lado, a corrente positivista defende essa aproximação, ao focar-se no teste de hipóteses, na utilização de métodos quantitativos e na estimação estatística de modelos; e, por outro, a corrente pós-positivista advoga a diferenciação metodológica, ao defender estratégias metodológicas qualitativas que permitam a compreensão dos significados, visando proceder à análise das subjetividades, da ambiguidade e das interpretações.

Ao debate entre positivismo e pós-positivismo, que caracteriza, de certo modo, todas as ciências sociais, aduz-se ainda a dificuldade de as ciências sociais, no geral e a ciência das políticas públicas, em particular, poderem ter objetivos que não se cingem aos de descrição e análise, envolvendo também objetivos de prescrição e 
6. Sobre a evolução do estudo das designadas redes de governação, veja-se, entre outros, Mota \& Bittencourt (2019).

7. Em sua abordagem da "sociologia da ação pública", Pierre Lascoumes e Patrick Le Galès (2007) vão ao encontro de muitas das recomendações apresentadas para o presente da ciência das políticas públicas, ao advogar uma ciência aplicada, recusar uma epistemologia particular e adotando uma perspetiva pluralista e pluricêntrica da atuação de múltiplos atores públicos e sociais no processo de políticas públicas e de governança pública. transformação (Ayres \& Marsh, 2013). Será importante recordar, a este propósito, que Lasswell advogava, precisamente, a utilização do conhecimento científico em prol de uma tomada de decisão política mais informada e esclarecida como um dos princípios orientadores da designada "policy orientation". No entanto, o apelo de aplicabilidade do conhecimento não tem sido totalmente bem acolhido, não só por parte dos académicos, que consideram que isso pode desvirtuar e corromper o conhecimento científico, como também por parte dos políticos, que frequentemente consideram que os académicos têm um conhecimento demasiado teórico e que os processos de investigação não se coadunam com os propósitos e timings da vida política (Cairney \& Weible, 2017). A este propósito, Cairney e Weible (2017) fazem um apelo para o desenvolvimento de uma nova abordagem à "ciência das políticas públicas" que incorpore a análise de políticas públicas e a investigação sobre o processo político. Para estes autores, esta nova abordagem deverá agregar a ciência básica e a ciência aplicada, tomar atenção aos contextos e dedicar maior atenção aos vários processos e atores de tomada de decisão, que não se cingem ao momento da formulação e aos atores formais (Cairney \& Weible, 2017).

Para além dos debates referidos, o presente e o futuro da ciência das políticas públicas é ainda marcado pela constatação da crescente complexificação dos problemas e das estratégias de governação pública (DeLeon \& Martell, 2006; Knill \& Tosun, 2020). Em face destas constatações, o hibridismo dos modelos, a mudança e a aprendizagem têm-se constituído como importantes enfoques temáticos de investigação (Knill \& Tosun, 2020). Na esfera analítica, tem-se assistido a uma maior atenção a fatores contextuais a partir de taxonomias contingenciais, a estudos comparativos, bem como a técnicas de análise participativas e de relacionamento em rede $^{6}$, as quais abrem novas perspetivas de recurso à abordagem pós-positivista e de maior aplicabilidade7 (DeLeon \& Martell, 2006; Ayres \& Marsh, 2013).

O apelo a uma maior tolerância teórica e empírica quanto às estratégias de investigação, bem como a escolha da estratégia analítica de acordo com o problema a investigar, constituem-se, assim, as principais invocações na ciência das políticas públicas da atualidade (Ayres \& Marsh, 2013). No entanto, permanece a dúvida sobre se o ecletismo continuará a ser uma das principais características desta disciplina ou se, pelo contrário, a direção futura será o desenvolvimento de um paradigma mais uniforme em face da ainda predominância do positivismo (Peters \& Pierre, 2006). Tendo em atenção os ensinamentos de Thomas Kuhn e Robert Merton sobre filosofia da ciência, considera-se que a existência de diversas abordagens analíticas de "médio alcance" sobre uma mesma realidade social é um sinal de maturidade disciplinar muito possivelmente inevitável. 
Diante do exposto, poder-se-á concluir que a ciência das políticas públicas se encontra, na atualidade, em processo de transição e que, apesar dos avanços que se têm registado desde a origem desta disciplina, permanecem muitos desafios a ultrapassar.

\section{Referências}

AYRES, S.; MARSH, A. Reflections on contemporary debates in policy studies. Policy \& Politics, v. 41, n. 4, p. 643-663, 2013. Disponível em: <doi:https://doi.org/10.1332/ $147084413 \times 674001>$.

BAUMGARTNER, F.; JONES, B. Agendas and instability in American politics. Chicago, IL: University of Chicago Press, 1993.

BAUMGARTNER, F.; JONES, B.; MORTENSEN, P. Punctuated equilibrium theory: explaining stability and change in public policymaking. In: WEIBLE, C.; SABATIER, P. Theories of the Policy Process, 4. ed., p. 55-102. New York: Routledge, 2018. Disponível em: <doi:https://doi.org/10.4324/9780429494284-3>.

CAIRNEY, P.; WEIBLE, C. The new policy sciences: combining the cognitive science of choice, multiple theories of context, and basic and applied analysis. Policy Sciences, v. 50, p. 619-627, 2017. Disponível em: <doi:https://doi.org/10.1007/ s11077-017-9304-2>.

DELEON, P.; MARTELL, C. R. The policy sciences: past, present, and future. In: PETERS, B.; PIERRE, J. Handbook of public policy, p. 31-47. London: Sage, 2006. Disponível em: <doi:http://dx.doi.org/10.4135/9781848608054>.

DELEON, P.; VOGENBECK, D. M. Back to square one: the history and promise of the policy sciences. In: RABIN, J.; HILDRETH, W.; MILLER, G. Handbook of public administration, 3. ed., p. 503-539. Boca Raton, FL: CRC Press, 2007. Disponível em: <doi:https://doi.org/10.4324/9781315093215-13>.

EASTON, D. A system analysis of political life. New York: Wiley, 1965.

FARAH, M. Análise de políticas públicas no Brasil: de uma prática não nomeada à institucionalização do "campo de públicas". Revista de Administração Pública, v. 50, n. 6, p. 959-979, 2016. Disponível em: <doi:https://doi.org/10.1590/0034$7612150981>$

HALL, P. (). Policy paradigms, social learning, and the State: the case of economic policymaking in Britain. Comparative Politics, v. 25, n. 3, p. 275-296, 1993. Disponível em: <doi:https://doi.org/10.2307/422246>. 
HEIKKILA, T.; CAIRNEY, P. Comparison of theories of the policy process. In: WEIBLE, C.; SABATIER, P. Theories of the policy process, 4. ed., p. 301-328. New York: Routledge, 2018. Disponível em: <doi:https://doi.org/10.4324/9780429494284>.

HILL, M. The sociology of public administration. London: Heinemann, 1972.

HILL, M.; HUPE, P. Implementing public policy. 3. ed. London: Sage, 2014. Disponível em: <https://uk.sagepub.com/en-gb/eur/implementing-public-policy/book240256 \#contents $>$.

HJERN, B.; HULL, C. Implementation research as empirical constitutionalism. European Journal of Political Research, v. 10, n. 2, p. 105-115, 1982.

HJERN, B.; PORTER, D. Implementation structures: a new unit of administrative analysis. Organization Studies, v. 2, n. 3, p. 211-227, 1981.

HOOD, C. The tools of government. Chatham, UK: Chatham House, 1986.

HOWLETT, M.; RAMESH, M.; PERL, A. Studying public policy: principles and processes. 4. ed. Toronto, CA: Oxford University Press, 2020. Disponível em: <https:// global.oup.com/academic/product/studying-public-policy-9780199026142?q=Michael\%20Howlett\&lang=en\&cc=pt\#>.

JANN, W.; WEGRICH, K. Theories of the policy cycle. In: FISCHER, F.; MILLER, G.; SIDNEY, M. Handbook of public policy analysis: theory, politics, and methods, p. 43-62. Boca Raton, FL: CRC Press, 2007. Disponível em: <doi:https://doi. org/10.4324/9781315093192>.

JENKINS-SMITH, H.; NOHRSTEDT, D.; WEIBLE, C.: INGOLD, K. Advocacy coalition framework: an overview of the research program. In: WEIBLE, C. Theories of the policy process. 4. ed. p. 135-172. New York: Routledge, 2018. Disponível em: <doi:https://doi.org/10.4324/9780429494284>.

JOHN, P. Analyzing public policy. 2. ed. Abingdon, UK: Routledge, 2013. Disponível em: <doi:https://doi.org/10.4324/9780203136218>.

KINGDON, J. Agendas, alternatives, and public policies. 2. ed. New York: Pearson, 2010. Disponível em: <https://www.pearson.com/us/higher-education/program/ Kingdon-Agendas-Alternatives-and-Public-Policies-Update-Edition-with-an-Epilogue-on-Health-Care-2nd-Edition/PGM95175.html?tab=overview>.

KNILL, C.; TOSUN, J. Public policy: a new introduction. 2. ed. New York: Palgrave MacMillan, 2020. Disponível em: <https://www.macmillanihe.com/page/detail/public-policy-christoph-knill/?k=9780230278387>. 
LASCOUMES, P.; LE GALÈS, P. Sociologie de l'action publique. Paris: Armand Colin, 2007.

LASSWELL, H. The policy orientation. In: LERNER, D.; LASSWELL, H. The policy sciences: recent developments in scope and method, p. 3-15. Stanford, CA: Stanford University Press, 1951.

MOTA, L. Estudos de implementação de políticas públicas: uma revisão de literatura. Sociologia, Problemas e Práticas, v. 92, p. 133-150, 2020. Disponível em: <doi:https://doi.org/10.7458/SPP20209211728>.

MOTA, L.; BITTENCOURT, B. Governação pública em rede: contributos para a sua compreensão e análise (em Portugal e no Brasil). Tempo Social, v. 31, n. 2, p. 199-219, 2019. Disponível em: <doi:https://doi.org/10.11606/0103-2070.ts.2019.147567>.

OSTROM, E. Institutional rational choice: an assessment of the institutional analysis and development framework. In: SABATIER, P. Theories of the policy process, p. 21-64. Boulder, CO: Westview Press, 2007. Disponível em: <doi:https://doi. org/10.4324/9780367274689-2>.

PARSONS, W. Public policy: an introduction to the theory and practice of policy analysis. Aldershot, UK: Edward Elgar, 1995. Disponível em: <https://www.e-elgar. com/shop/gbp/public-policy-9781852785543.html>.

PETERS, B.; PIERRE, J. Introduction. In: PETERS, B.; PIERRE, J. (Eds.). Handbook of public policy, p. 1-9. London: Sage, 2006. Disponível em: <doi:http://dx.doi. org/10.4135/9781848608054>.

PIERRE, J. Disciplinary perspectives. In: PETERS, B.; PIERRE, J. (Eds.). Handbook of public policy, p. 1-9. London: Sage, 2006. Disponível em: <doi:http://dx.doi. org/10.4135/9781848608054>.

SABATIER, P. The need for better theories. In: Sabatier, P. Theories of the policy process, p. 3-17. Boulder, CO: Westview Press, 2007. Disponível em: <doi:https://doi. org/10.4324/9780367274689-2>.

Political science and public policy. PS: Political Science and Politics, v. 24, n. 2, p, 144-147, 1991. Disponível em: <doi:https://doi.org/10.2307/419922>.

SABATIER, P.; JENKINS-SMITH, H. (Eds.). Policy change and learning: an advocacy coalition approach. Boulder, CO: Westview Press, 1993.

SABATIER, P.; WEIBLE, C. Theories of the policy process. 3. ed. Boulder, CO: Westview Press, 2014. 
SCHARPF, F. Interorganizational policy studies: issues, concepts, and perspectives. In: HANF, K.; SCHARPF, F. Interorganizational policy making: limits to coordination and central control. London: Sage, 1978.

TAVARES, A. A análise de políticas públicas como profissão: retratos de uma infância difícil. In: TAVARES, A. Estudo e ensino da administração pública em Portugal, p. 83-100. Lisboa: Escolar Editora, 2006. Disponível em: <https://escolareditora.com/ store/product/0/2444/estudo-e-ensino-da-administracao-publica-em-portugal>.

WEIBLE, C.; SABATIER, P. Theories of the policy process. 4. ed. New York: Routledge, 2018. Disponível em: <doi:https://doi.org/10.4324/9780429494284>. 